Charles Nrcolte. Dastin des maladies infectieuses.

LECoQ. Déséquilibres alimentaire, Paris, 1938.

(Travail du Service de documentation de la Fabrique suisse des produits au lait Guigoz S. A. Vuadens (Gruyère)).

\title{
LA RÉGLEMENTATION DU LAIT MALPROPRE
}

\author{
par \\ L. HOTON, \\ Docteur ès sciences.
}

Nous sommes tous d'accord sur un point : le lait doit être pur, sain, propre. Laissons de côté les questions relatives à la falsification du lait, mais admettons que si un lait sain est toujours un lait propre, la réciproque n'est pas toujours vraie : la propreté d'un lait, celle qui correspondrait aux exigences d'une réglementation, n'est pas nécessairement le corollaire de la salubrité.

Est-il utile de fixer, par voie de décret, des normes ou des bases analytiques permettant à l'expert de déclarer que tel lait est ou non conforme aux stipulations réglementaires ?

A mon avis, non. La réglementation doit rester, comme on l'a fait en France, dans des généralités; elle doit se borner à exiger que le lait soit recueilli proprement, et qu'il ne soit pas permis de le vendre s'il est coloré, malpropre ou malodorant.

J'exposerai plus loin les raisons qui montrent les dangers d'une définition légale de la propreté du lait.

$\mathrm{Si}$ on parcourt les législations relatives au lait, on constate que sauf une seule exception, les dispositions restent dans les généralités.

L'exception concerne la Belgique. Ce pays avait primitivement édicté en 1894 un règlement qui, à mon sens, était parfait : "il défendait la mise en vente du lait altéré soit..., soit par une tenue défectueuse de l'étable, de la laiterie ou des ustensiles de transport, soit pour toute autre cause telles les manipulations exercées par des personnes peu soigneuses."

Ce règlement est resté en vigueur jusqu'en 1925, c'est alors qu'on a cru devoir indiquer aux experts les eas de souillure qui légalement rendaient le vendeur justiciable des tribunaux.

La Suisse défendait la vente "du lait qui forme un sédiment (1908) \%. Le projet français de 1912 est muet en ce qui concerne la propreté.

En 1915, la Suisse précisait la défense de 1908 en ces termes : "Le lait sera recueilli avec tous les soins de propreté possibles. Destiné à être consommé tel quel, il ne doit pas laisser déposer, 
par le repos, des quantités nettement appréciables d'impuretés $x$. Le décret français du 25 mars 1924 défend par son article 2, § 3 , la vente " de lait coloré, malpropre ou malodorant ".

La définition arrêtée au Congrès de la Répression des Fraudes de 1909 est précisément celle reprise par le décret français : On considère comme impropre à la consommation le lait coloré, malpropre, malodorant.

Et on constate, dès la mise en vigueur de ces dispositions si brèves, mais en même temps si précises, que les tribunaux les appliquent sans les entourer d'explications, de commentaires et de "considérant " plus ou moins obscurs.

Le Tribunal correctionnel de la Seine (30 septembre 1926) punit un laitier vendant du lait sale, impropre à la consommation.

Celui de Versailles (23 mars 1928) condamne la vente d'un lait u a lifié de malpropre.

Celui de Tarbes (16 mars 1928) rend un même jugement : vente de lait sale, impropre à la consommation.

Même jugement au Tribunal de l'Aube (6 avril 1928) : vente d'un lait malpropre.

En sa séance du 9 novembre 1927, la Société des Experts chimistes de France avait entendu un travail de M. Fouassier relatif à l'identification des laits sales. La question n'a, du moins je le pense, plus été soulevée.

La circulaire interprétative du 25 septembre 1924, § 1, spécifie que "le lait-sera recueilli proprement ".

En 1929, les Etats-Unis définissent le lait « la sécrétion entière, fraîche, propre ".

L'Italie (1931) ordonne la filtration du lait et défend (1932) la mise en vente d'un lait qui forme un sédiment après un quart d'heure de repos.

L'Allemagne se contente de décréter : "Le lait sera protégé pendant et après la récolte " (1933).

La Tchécoslovaquie (1937) défend la vente du lait qui donne un sédiment après un quart d'heure.

Voici, in fine, le texte de l'arrêté belge ; il est antérieur aux arrêtés des Etats-Unis, de l'Allemagne et de la Tchécoslovaquie. L'article 15, $\$ 8$ (31 mars 1925), défend la mise en vente. de lait souillé, par des impuretés telles que parcelles de fourrages ou d'excréments, poils, mouches, débris de matières organiques, qui donne après 4 heures au moins de repos, un sédiment de $0 \mathrm{~cm} / 5$ d'impuretés par demi-litre de lait, ou qui contient par litre plus de 12 milligrammes d'impuretés insolubles dans l'eau, l'alcool, l'éther, sauf à prouver par une épreuve à la fermentation ou par une analyse bactériologique l'absence de microbes de la putréfaction ". 
Un nouvel arrêté pris un an après est venu très justement supprimer la dernière phrase. Et en effet, on pouvait, à première lecture, se demander a priori à qui incombait la charge de la preuve? Est-ce au vendeur du lait à établir que sa marchandise ne contenait pas de microbes de la putréfaction ? Est-ce au service d'inspection ? La réponse n'était cependant pas douteuse ; cette preuve incombait, judiciairement parlant, à la partie poursuivante. C'est, en matière de procédure, dénommé le fardeau de la preuve.

Il suffisait de réfléchir un peu pour se rendre compte que ce malencontreux paragraphe rendait la mise en pratique des stipulations qui le précédaient difficiles sinon impossibles dans la pratique.

$$
* * *
$$

La question qui se pose est celle-ci : Ies laits qui répondent aux conditions arrêtées par la Belgique, la Suisse, la Tchécoslovaquie peuvent-ils rentrer dans la catégorie des " non malpropres " ? Est-ce que l'épreuve qu'ils ont subie leur confère les mérites du lait propre-? Cent fois non! Tous les techniciens seront d'accord avec moi pour dire qu'à côté de ce que l'on voit, il y a ce qui reste caché, que l'épreuve de filtration ou de sédimentation nous montre les souillures tangibles, mais qu'à côté de ces dernières, peuvent très bien se trouver les germes d'infection, microbes, bacilles, spores de toute nature qui filtreront complètement au travers des mailles les plus serrées, ou qui ne donneront jamais lieu à la moindre sédimentation.

Un chimiste américain a démontré que le lait s'infecte surtout par les bouses de vache liquides ou semi-liquides et que ces germes de l'infection traversent les filtres les plus serrés. Il concluait que ces laits infectés pouvaient être bien plus dangereux que ceux souillés par les matières solides... Conclusion toute naturelle et qui pouvait se postuler sans être appuyée d'expériences de laboratoire.

J'ai assisté à quelques expériences d'essai de filtration des laits d'un même troupeau, et ultérieurement, j'ai personnellement continué ces expériences.

Les résidus des filtres sont différents si la traite s'effectue à l'étable même, ou dans un local voisin, ou à la prairie. Ils sont différents si les pis sont lavés, si l'opérateur revêt une blouse propre, etc. ; si les laits sont recueillis à la prairie, on observera des différences notables entre les traites effectuées par temps calme, par vent violent, ou immédiatement après une pluie. Encore ici, les résultats sont prévisibles.

Malgré la confiance fort limitée que j'ai dans l'épreuve officielle de filtration imposée par l'arrêté belge, j'estime eependant qu'elle présente son utilité car elle oblige le producteur et le débitant 
à filtrer soigneusement le lait et à en écarter ainsi les souillures visibles. Qu'on me permette d'utiliser l'expression vulgaire : c'est autant de pris sur l'ennemi.

Mais n'exagérons pas, comme certains l'ont fait, les mérites, l'efficaeité de la filtration.

Lors de l'étude de l'arrêté belge de 1925, le personnel du service d'inspection a été tenu à l'écart de la Commission chargée de présenter les modifications à l'ancienne réglementation. Les suggestions formulées par les inspecteurs principaux placés à la tête des quatre circonscriptions du Royaume ont été transmises à la Commission et celle-ci n'en a tenu aucun compte.

Des services municipaux n'appartenant pas à l'Inspection des denrées ressortissant au Ministère de l'Intérieur (Administration de l'Hygiène) avaient, dès 1921, entrepris, dans quelques localités, une campagne tendant à rendre la filtration du lait obligatoire, but louable en soi, mais, dans leur désir de trop prouver, les agents exagérèrent! C'est ainsi qu'après avoir, lors d'une première tournée, recueilli des laits en vue de l'essai de filtration, ils revenaient sur les lieux avec les rondelles filtrantes, surchargées d'impuretés. Les autorités communales (municipales), frappées par la saleté des laits, ordonnaient alors la filtration. Les mêmes agents recommençaient leurs opérations et on constatait, cette fois, une amélioration notable et parfois totale.

Rien à critiquer jusqu'ici, mais là où " la mesure était dépassée ", c'est quand, comme je l'ai vu dans la banlieue de Liége, on affichait à la porte des écoles les deux séries de filtres sous les rubriques : "Avant l'inspection : laits contaminés ! " et "Après l'inspection : laits sains!"

Qualifier des laits simplement filtrés "laits sains ", c'est ou de l'aberration scientifique ou du cynisme.

Cette campagne prit fin à la suite des protestations légitimes du service officiel d'inspection. Mais ces protestations n'eurent aucun effet sur les membres de la Commission chargée de l'étude de la nouvelle réglementation. Je suis même tenté de dire $\&$ au contraire " ! On fit passer sous leurs yeux de nombreux filtres dits "Avant et après ". Certains des filtres "Avant " avaient été soigneusement souillés d'impuretés qu'on ne rencontre jamais dans le lait. Comme argument, si e'était convaincant, disons aussi que c'était peu honnête !

Quels sont les résultats en Belgique des prescriptions réglementaires ? La filtration qui avait toujours été pratiquée antérieurement à 1925 , continue à s'effectuer, mais les producteurs y apportent, en général, plus de soins, les mailles des filtres sont maintenant 
plus serrées qu'avant ; c'est là le résultat le plus sensible obtenu... peu de choses en somme.

A côté du service d'inspection gouvernemental, de nombreuses communes ont institué un service de contrôle municipal ; certains de ces services attachent la plus grande importance, je suis tenté d'écrire "trop d'importance " à l'essai de filtration et continuent à colporter, à afficher et à mettre en vedette dans des expositions le test de filtration "Avant l'inspection et après l'inspection ". Nous avons depuis quelques mois une " commission du lait $n$ comprenant toutes les compétences belges en matière de production laitière. S'occupera-t-elle de reviser les dispositions réglementaires que j'ai étudiées plus haut?

Comme je l'ai dit, la filtration doit être rendue obligatoire. C'est une question primordiale, mais il serait nécessaire d'avertir les consommateurs, aussi bien que les producteurs, que cette mesure $n^{\prime}$ a pas pour effet de transformer un lait infecté en un produit parfait.

Il est toujours dangereux d'entrer dans trop de détails, de vouloir trop préciser en matière de réglementation; le texte légal belge de 1894 n'aurait pas dû être modifié ; si on tenait absolument à le changer, on aurait pu adopter la rédaction française de 1924 qui spécifie, sans plus, que le lait sera recueilli proprement et qu'il ne sera ni coloré, ni malpropre, ni malodorant.

C'est clair et net, et les tribunaux ont ainsi une base solide pour la répression, l'exemple des jugements prononcés en France nous en donne la preuve.

\title{
UNE MÉTHODE RAPIDE POUR L'ESTIMATION DES PROPRIÉTÉS ÉLASTIQUES ET PLASTIQUES de la caséine a la présure
}

\author{
par \\ G. GENIN \\ Ingénieur Chimiste E. P. C.
}

On sait l'importance que présente la caséine en tant que matière première pour la fabrication de certaines matières plastiques, constituées essentiellement de caséine durcie à la formaldéhyde et qui sont connues en particulier sous le nom de galalith.

Il est certain que l'utilisation de ta caséine pour la fabrication de ces produits et l'emploi de ces derniers se sont trouvés limités du fait de la variabilité de la matière première utilisée. Autrement dit, lorsque dans une usine, on utilise des lots successifs de caséine à la présure pour fabriquer de la galalith ou toute substance analogue, on est parfois dans l'obligation d'apporter certaines modifications 